\title{
LILRB2 Gene
}

National Cancer Institute

\section{Source}

National Cancer Institute. LILRB2 Gene. NCI Thesaurus. Code C102793.

This gene is involved in immunity. 